\title{
Aberrant promoter DNA methylation inhibits bone morphogenetic protein 2 expression and contributes to drug resistance in breast cancer
}

\author{
MIN DU ${ }^{1}$, XIAO-MEI SU ${ }^{1}$, TAO ZHANG ${ }^{1}$ and YONG-JUN XING ${ }^{2}$ \\ ${ }^{1}$ Department of Oncology and ${ }^{2}$ Affiliated Stomatological Hospital, PLA General Hospital of Chengdu Military Region, \\ Chengdu, Sichuan 610083, P.R. China
}

Received September 12, 2013; Accepted March 17, 2014

DOI: $10.3892 / \mathrm{mmr} .2014 .2276$

\begin{abstract}
Bone morphogenetic protein 2 (BMP2) is a growth factor that is involved in the development and progression of various types of cancer. However, the epigenetic regulation of the expression of BMP2 and the association between BMP2 expression and drug resistance in breast cancer remains to be elucidated. The present study reported that the expression of BMP2 was significantly decreased in primary breast cancer samples and the MCF-7/ADR breast cancer mulitdrug resistance cell line, which was closely associated with its promoter DNA methylation status. The expression of BMP2 in MCF-7/ADR cells markedly increased when treated with 5-Aza-2'-deoxycytidine. Knockdown of BMP2 by specific small interfering RNA enhanced the chemoresistance of the MCF-7 breast cancer cell line. These findings indicated that epigenetic silencing of BMP2 in breast cancer may be involved in breast cancer progression and drug resistance, and provided a novel prognostic marker and therapeutic strategy for breast cancer.
\end{abstract}

\section{Introduction}

Breast cancer is one of the most common types of cancer among females (1). Although progress has been made in diagnosis and adjuvant therapies, the pathogenesis of breast cancer remains unknown. Furthermore, drug resistance also remains a major clinical obstacle for the successful treatment of breast cancer. Bone morphogenetic proteins (BMPs), belonging to the TGF- $\beta$ superfamily, had been confirmed in several studies to be important in cell proliferation, differentiation and apoptosis during tumor progression (2-4). In breast cancer, it was

Correspondence to: Dr Yong-Jun Xing, Affiliated Stomatological Hospital, PLA General Hospital of Chengdu Military Region, No. 270 Rongdu Road, Chengdu, Sichuan 610083, P.R. China

E-mail: yongjunxing@gmail.com

Key words: DNA methylation, bone morphogenetic protein 2, breast cancer, drug resistance revealed that BMP2, BMP4, BMP6, BMP7, BMP9 and BMP10 were involved in breast cancer development, progression and drug resistance (5-10). However, few studies focusing on the epigenetic regulation of the expression of BMPs have been conducted. Recently, it was recognized that DNA methylation was a common event in human cancer, which induced the loss of tumor suppressor gene expression (11-12). Previous studies have also demonstrated that the downregulation of BMP2 was silenced by aberrant DNA methylation in bone formation and gastric carcinomas $(13,14)$. To date, the epigenetic regulation of BMP2 in breast cancer and its association with tumor progression and drug resistance remains to be elucidated.

The present study detected the mRNA expression and the promoter methylation status of BMP2 in breast cancer tissues and breast cancer cell lines. In addition, BMP 2 expression and cancer cell chemoresistance was also evaluated in an in vitro cell model. Our data indicated that the downregulation of BMP2 caused by promoter hypermethylation may contribute to breast cancer progression and cancer cell drug resistance.

\section{Materials and methods}

Patients and tissue specimens. A total of 32 breast cancer tissues and patient-matched adjacent normal breast tissues were obtained from patients who underwent surgery at the Cancer Hospital of Sichuan Province (Chengdu, China). All tumor tissues were reviewed by an experienced pathologist using World Health Organization recommendations on histopathological typing. For total RNA isolation, the specimens were frozen in liquid nitrogen and stored at $-80^{\circ} \mathrm{C}$. There were no patients who received chemotherapy or radiotherapy prior to surgery. The study protocol conformed to the local ethical standards of the institutional review board of the The PLA General Hospital of Chengdu Military Region (Chengdu, Sichuan, China).

Cell culture. The MCF-7 human breast cancer cell line was purchased from the Shanghai Institute for Biological Sciences, Chinese Academy of Sciences (Shanghai, China) and MCF-7/ADR, a doxorubicin resistant subline of MCF-7, was generated by continuously culturing the drug-sensitive parental cell line MCF-7 in medium containing incrementally 
increasing concentrations of doxorubicin (Sigma-Aldrich, St. Louis, MO, USA). The cells were cultured in RPMI-1640 (Gibco-BRL, Carlsbad, CA, USA) supplemented with $10 \%$ fetal calf serum, $100 \mathrm{U} / \mathrm{ml}$ penicillin and $100 \mathrm{U} / \mathrm{ml}$ streptomycin at $37^{\circ} \mathrm{C}$ and $5 \% \mathrm{CO}_{2}$. To avoid the effects of the drug, the MCF-7/ADR drug resistant cell line was cultured in drug-free medium for $>2$ weeks prior to subsequent experiments.

Treatment of cells with 5-aza-2'-deoxycytidine (5-aza-dc). The cells were seeded at a density of $1 \times 10^{5}$ cells in 6 -well plates. Following $24 \mathrm{~h}$, the cells were treated with $5 \mu \mathrm{M}$ 5-aza-dc (Sigma-Aldrich). Total cellular RNA and protein were isolated from the cells 0 and 3 days after the addition of 5-aza-dc as described above.

RNA isolation and quantitative polymerase chain reaction ( $q P C R$ ). The total RNA of 32 cancer and adjacent normal breast tissues were extracted using TRIzol reagent (Invitrogen Life Technologies, Carlsbad, CA, USA) according to the manufacturer's instructions. Total RNA was retrotranscribed using the RevertAid ${ }^{\mathrm{TM}}$ First Strand cDNA Synthesis kit (Thermo Fisher Scientific, Waltham, MA, USA) according to the manufacturer's instructions. qPCR was performed with the SYBR-Green PCR master mix (Takara Bio, Inc., Shiga, Japan) using a Mastercycler ep realplex (Eppendorf, Hamburg, Germany). The levels of mRNA expression were quantified following normalization with endogenous control GAPDH using the $2^{-\Delta \Delta C T}$ method (15). The primer sequences used for PCR were as follows: Forward: 5'-CTTTGGTATCGTGGAAGGACTC-3' and reverse: 5'-GTAGAGGCAGGGATGATGTTCT-3' for GAPDH (product size: $132 \mathrm{bp}$ ); and forward: 5'-CCCAGCGTGAAAAGAGAGAC-3' and reverse: 5'-GAGACCGCAGTCCGTCTAAG-3' for bmp2 (product size: $168 \mathrm{bp}$ ). The experiments were performed independently for each sample and at least three technical replicates were run for each treated sample and controls.

Western blot analysis. Whole cell extracts were lysed using lysis buffer pulsed protease inhibitor (Millipore, Billerica, MA, USA). Equivalent quantities of protein were resolved by SDS-PAGE, transferred onto polyvinylidene difluoride membranes (GE Healthcare Biosciences, Piscataway, NJ, USA), inhibited with phosphate-buffered saline (PBS) containing $0.2 \%$ Tween-20 and 5\% non-fat dry milk and incubated with primary antibodies against BMP2 $(1: 1,000$; rabbit polyclonal to $\mathrm{BMP} 2)$ and actin $(1: 2,000$, mouse monoclonal to actin) obtained from Abcam (Cambridge, MA, USA). Antibody binding was revealed by incubation with horseradish peroxidase-conjugated secondary antibodies (Invitrogen Life Technologies) and an Enhanced Chemiluminescnce Plus immunoblotting detection system (GE Healthcare Biosciences). The signals were quantified using NIH Image J 1.63 software (National Institutes of Health, Bethesda, MA, USA).

Methylation-specific PCR (MSP) and bisulfite genomic sequencing. Genomic DNA of MCF-7 or MCF-7/ADR was extracted using a TIANamp Genomic DNA kit (Tiangen
Biotech (Beijing) Co., Ltd., Beijing, China). Bisulfite conversion of genomic DNA was performed using the EZ DNA Methylation-Gold kit (Zymo Research, Irvine, CA, USA) according to the manufacturer's instructions. MSP was performed on bisulfite-modified DNA. The primer sequences of BMP-2 for the unmethylated reaction were: Forward: 5'-GGGTTAGTGTTGAGTGGATTATT-3' and reverse: 5'-CACAACACTAAAAATCAACTCC-3' and for the methylated reaction forward: 5'-TTAGCGTCGAGTGGATTATC-3' and reverse: 5'-GACGCTAAAAATCGACTCC-3'. PCR products were analyzed by electrophoresis in a $2.0 \%$ agarose gel containing golden view (SBS Genetech Co., Ltd., Beijing, China). For bisulfite sequencing of DNA samples, sodiumbisulfite-treated genomic DNA was amplified using the following primers: Forward: 5'-GTGGTTTTTGTTGTTYGG-3' and Reverse: 5'-TCTACCTTACTCCAATACACCC-3'. The PCR reaction products were gel purified and cloned into the pGEM-T Vector system according the manufacturer's instructions (Promega Corporation, Madison, WI, USA). The colonies were sequenced on a genetic analyzer (Applied Biosystems, Foster City, CA, USA) to analyze the methylated cytosine level.

Small interfering RNA (siRNA) transfection. siRNAs (Guangzhou RiboBio Co., Ltd., Guangzhou, Guangdong, China) were transfected using Lipofectamine ${ }^{\mathrm{TM}} 2000$ and OptiMEM (Invitrogen Life Technologies) according to the manufacturer's instructions. Following $72 \mathrm{~h}$ of siRNA transfection, the cell lysate was prepared and western blotting was performed as described above.

3-(4,5-dimethylthiazol-2-yl)-2,5-diphenyltetrazoliumbromide (MTT) assay. MCF-7 cells were seeded in 96-well plates at a density of $1 \times 10^{4}$ cells per well and incubated overnight in $10 \%$ fetal bovine serum medium. The cells were transfected with BMP2 specific siRNA or negative control siRNA. Then, the cells were treated with different concentrations of adriamycin (0-200 mg/l). Following incubation for $48 \mathrm{~h}$ at $37^{\circ} \mathrm{C}, 20 \mu \mathrm{l} \mathrm{MTT} \mathrm{(Sigma-Aldrich)} \mathrm{solution}(5 \mathrm{mg} / \mathrm{ml}$ in PBS) was added to each well and incubation continued for a further $4 \mathrm{~h}$ at $37^{\circ} \mathrm{C}$. The medium was then removed and the MTT crystals were solubilized using $100 \mu \mathrm{l}$ dimethylsulfoxide. Absorbance was measured at $560 \mathrm{~nm}$ using a microplate reader (Thermo Fisher Scientific). Absorbance readings were subtracted from the value of blank wells. The reduction in cell growth was calculated as a percentage of control absorbance in the absence of any drug. Data are presented as the mean \pm standard deviation (SD) of at least three independent experiments.

Statistical analysis. Data are expressed as the mean \pm SD. The paired t-test and independent sample t-test were applied for statistical analysis using SPSS software version 13.0 (SPSS, Inc., Chicago, IL, USA). $\mathrm{P}<0.05$ was considered to indicate a statistically significant difference.

\section{Results}

DNA methylation suppresses BMP2 expression in breast cancer tissues. The present study performed quantitative 
A

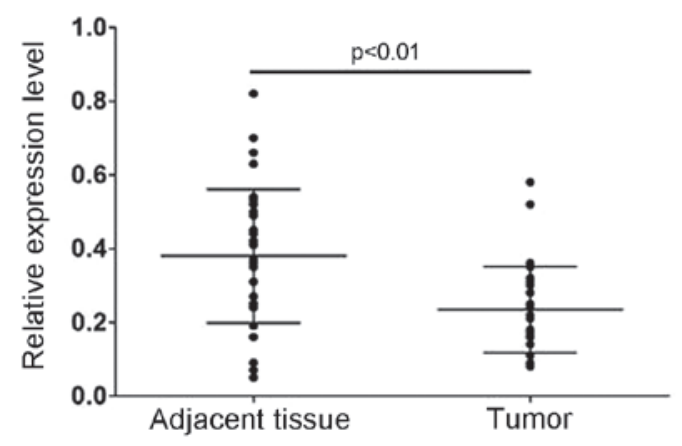

B

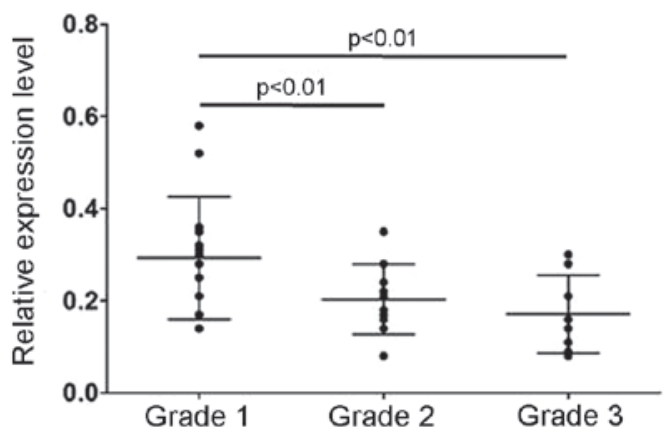

Figure 1. (A) Quantitative polymerase chain reaction demonstrated that the mRNA expression of BMP 2 was significantly decreased in breast cancer tissues compared with the adjacent normal tissues. (B) In tumor tissues, the BMP2 expression level was significantly higher in grade 1 tumors than in grade 2 and grade 3 tumors. BMP2, bone morphogenetic protein 2.

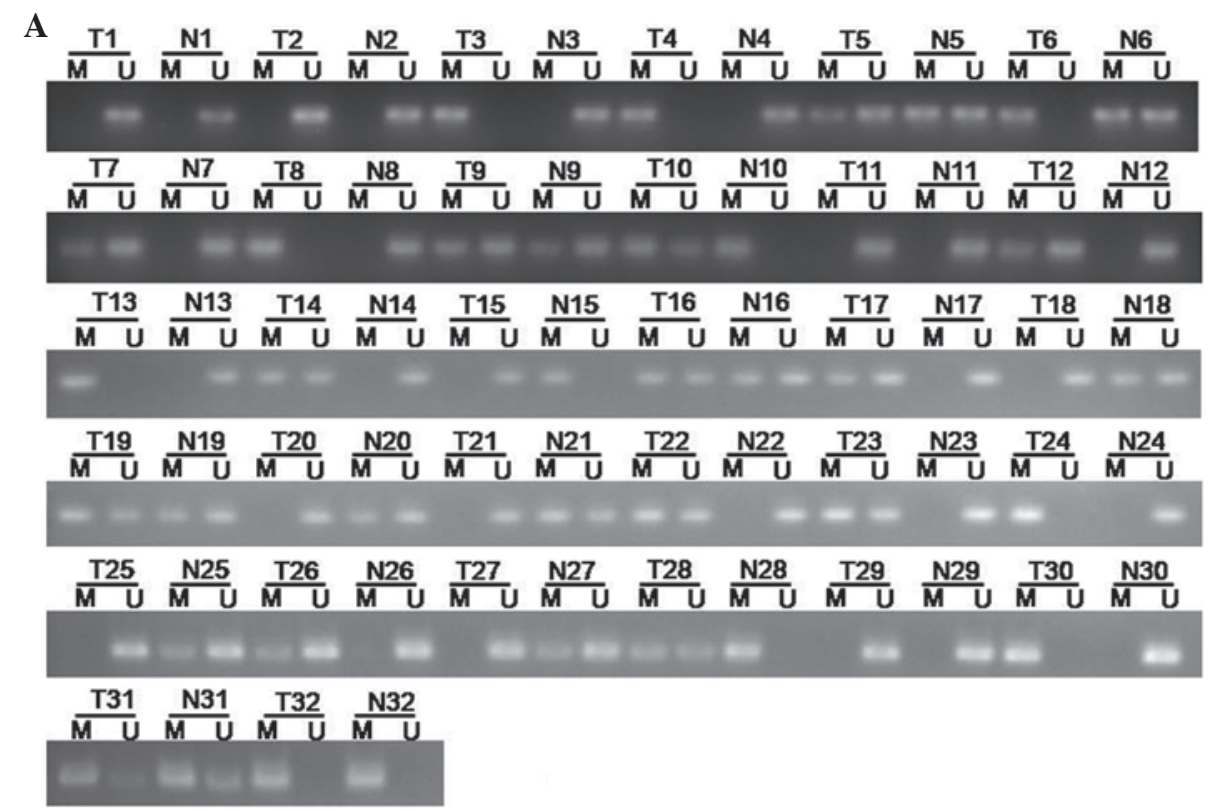

B

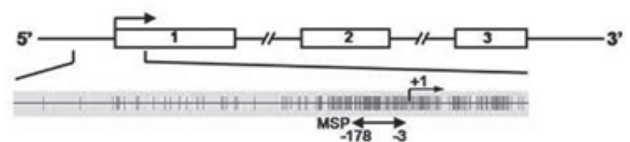

Figure 2. (A) MSP analyzed the BMP2 promoter methylation status in 32 breast cancer tissues and matched adjacent normal tissues. (B) Schematic diagram of the BMP2 promoter and the fragment for MSP analysis. BMP2, bone morphogenetic protein 2; MSP, methylation-specific polymerase chain reaction; M, methylated, U, unmethylated.

analysis of BMP2 mRNA in 32 breast cancer tissues and matched adjacent normal tissues by qPCR. Among the 32 pairs of samples, nine samples of breast cancer tissue demonstrated a higher expression of BMP2 compared with the adjacent normal tissue, and 23 samples of breast cancer tissue demonstrated a lower expression of BMP2 compared with the adjacent normal tissue. Compared with the normal tissues, BMP 2 mRNA expression was significantly lower in cancer tissues $(\mathrm{P}<0.01$; Fig. $1 \mathrm{~A})$. Furthermore, data demonstrated that the mRNA expression of BMP2 decreased between tumor stage 1 and stage 3 . There was a significantly higher level of BMP2 mRNA in stage 1 than in stage 2 and 3 tumors (Fig. 1B). Subsequently, the BMP2 methylation status was detected by MSP. Aberrant methylation was detected in 22 out of $32(68.75 \%)$ tumors, which was more frequent than that in the paired adjacent normal tissues [15 out of 32 (46.87\%); Fig. 2A].

DNA methylation suppresses the expression of BMP2 in drug resistant breast cancer cells. qPCR and western blot analysis demonstrated that BMP2 was expressed in the breast cancer cell line MCF-7 and was downregulated in doxorubicin resistant MCF-7/ADR cells (Fig. 3A and B). Following treatment with a DNA methyltransferase inhibitor 5-aza-dc, the downregulation of BMP2 was reversed in MCF-7/ADR cells (Fig. 3A and B). This indicated that the expression of BMP2 may be involved in DNA methylation regulation. Thus, MSP and bisulfite-sequencing PCR (BSP) were employed to investigate the promoter methylation status of BMP2. MSP analyses revealed that $\mathrm{CpG}$ islands 
A

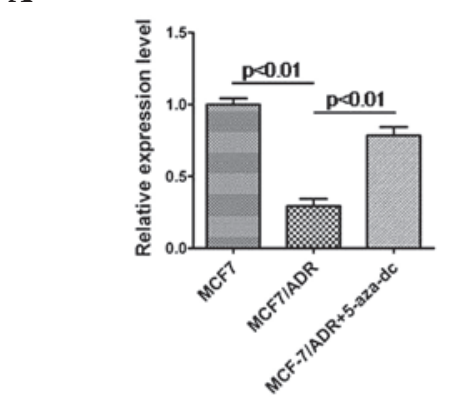

C

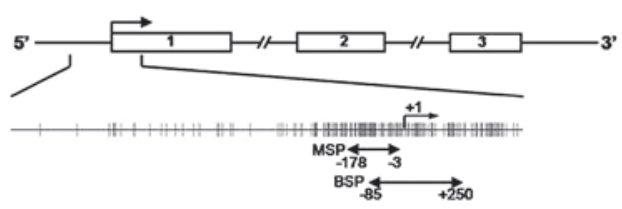

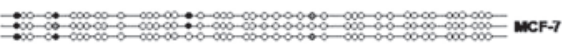

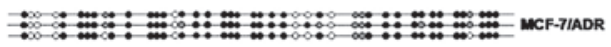

B

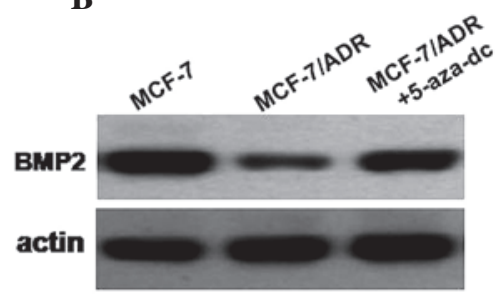

D

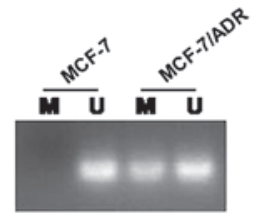

$\mathbf{E}$

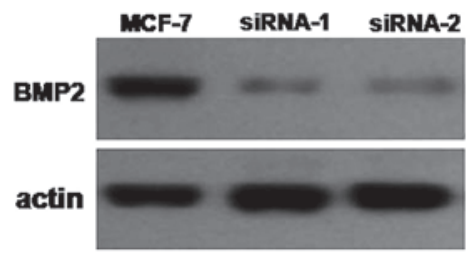

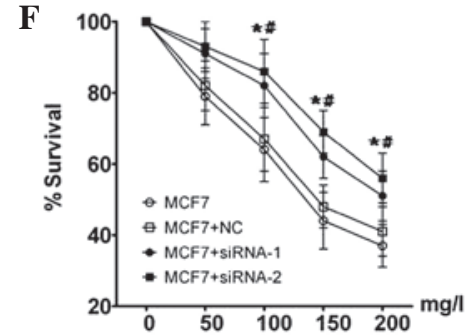

Figure 3. (A) Quantitative PCR and (B) western blot analysis demonstrated that BMP2 was downregulated in MCF-7/ADR drug resistant breast cancer cells and this downregulation may be reversed by treatment with 5-aza-dc. (C) Bisulfite-sequencing PCR and (D) methylation-specific PCR demonstrated an aberrant hypermethylation status of the BMP2 promoter. (E) Specific siRNA inhibited the expression of BMP2 and (F) enhanced the chemoresistance of MCF-7 breast cancer cells. "P<0.01 MCF-7 + siRNA-1 vs. MCF-7; ${ }^{\text {P }}<0.01 \mathrm{MCF}-7$ + siRNA-2 vs. MCF-7. BMP2, bone morphogenetic protein 2; 5-aza-dc, 5-aza-2'-deoxycytidine; siRNA, small interfering RNA; PCR, polymerase chain reaction; M, unmethylated; U, methylated.

of the BMP promoter were unmethylated in MCF-7 cells. However, in MCF-7/ADR cells, they were partially methylated (Fig. 3D). Furthermore the MSP results were confirmed by BSP. The results demonstrated that MCF-7/ADR cells showed dense methylation of the promoter $\mathrm{CpG}$ islands (Fig. 3C).

Knockdown of BMP2 by siRNA increases the chemoresistance of MCF-7 cells. In order to evaluate the effect of BMP2 downregulation in MCF-7 cells, the negative control siRNA and BMP2-specific siRNA were transfected into the MCF-7 cells and qPCR and western blot analysis detected the efficacy of the downregulation of expression of the BMP2 gene. The results indicated that siRNA was able to significantly downregulate the expression of BMP2 (Fig. 3E). Then, the MTT assay was employed to detect the effects of BMP2 expression downregulation by siRNA transfection on doxorubicin resistance. The survival ratio of $\mathrm{MCF}-7$ cells, MCF-7 cells transfected with negative control siRNA and BMP2-specific siRNA-1 and siRNA-2 were analyzed following treatment with a range of increasing concentrations of doxorubicin for $48 \mathrm{~h}$. The survival ratio of cells transfected with BMP2-specific siRNA-1 or siRNA-2 was significantly higher than cells transfected with negative control siRNA or MCF-7 cells (Fig. 3F). No significant difference between the MCF-7 cells and cells transfected with negative control siRNA was identified.

\section{Discussion}

The present study found that BMP2 expression was decreased in breast cancer tissues and was closely associated with DNA hypermethylation. Furthermore, the downregulation of BMP2 induced by DNA hypermethylation was able to enhance the chemoresistance of the MCF-7 breast cancer cell line. All these results indicated that aberrant methylation regulated BMP2 expression and was important in breast cancer development and drug resistance.

BMPs are a group of growth factors that belong to the TGF- $\beta$ superfamily. Previous studies confirmed that BMPs are important in cell proliferation, differentiation and apoptosis during development of the embryo and tumor progression (2-4). In breast cancer, the role of BMPs has not been well characterized, although several studies have focused on this subject $(16,17)$. BMP2 was reported to inhibit breast cancer cell proliferation through p21 and PTEN $(18,19)$. In addition, BMP2 may be involved in tumor angiogenesis, invasion and hormone-independent growth of breast cancer $(20,21)$. The data of the present study also confirmed that BMP2 was involved in breast cancer development and progression. However, there are few studies investigating the association between BMP2 and cancer cell drug resistance, and the mechanisms underlying the regulation of BMP2 expression. In the present study, the in vitro cell model revealed that the downregulation 
of BMP2 was able to enhance the chemoresistance of MCF-7 breast cancer cells. All these data indicated that BMP2 may be important in breast cancer development and drug resistance.

DNA methylation was recognized as one of the most common forms of gene expression regulation (22). In various types of human cancer, DNA methylation patterns are associated with cancer development and progression (23). In breast cancer, numerous studies have confirmed that DNA methylation is involved in tumorigenesis by inhibition of tumor suppressor gene expression, including estrogen receptor, hox5a, twist and E-cadherin (24-28). The present study revealed that DNA hypermethylation of BMP2 was closely associated with the downregulation of BMP2 in clinical breast cancer specimens. Furthermore, in an in vitro cell model, DNA hypermethylation led to the downregulation of BMP2 and resulted in enhanced chemoresistance of MCF-7 cells. These data indicted that DNA hypermethylation may cause breast cancer cell drug resistance through silencing BMP2 expression.

In conclusion, the results of the present study indicated that promoter hypermethylation suppression of BMP2 is a frequent event in human breast cancer. This aberrant epigenetic event may be important in the development and drug resistance of breast cancer, and our data may provide a novel prognostic marker and therapeutic strategy for breast cancer.

\section{References}

1. Siegel R, Naishadham D and Jemal A: Cancer statistics, 2013. CA Cancer J Clin 63: 11-30, 2013.

2. Slattery ML, John EM, Torres-Mejia G, et al: Genetic variation in bone morphogenetic proteins and breast cancer risk in hispanic and non-hispanic white women: The breast cancer health disparities study. Int J Cancer 132: 2928-2939, 2013.

3. Thawani JP, Wang AC, Than KD, et al: Bone morphogenetic proteins and cancer: review of the literature. Neurosurgery 66: 233-246, 2010.

4. Buijs JT, Petersen M, van der Horst G, et al: Bone morphogenetic proteins and its receptors; therapeutic targets in cancer progression and bone metastasis? Curr Pharm 16: 1291-1300, 2010.

5. Ye S, Park BH, Song KJ, et al: In vivo inhibition of bone morphogenetic protein-2 on breast cancer cell growth. Spine (Phila Pa 1976) 38: E143-E150, 2013.

6. Guo D, Huang J and Gong J: Bone morphogenetic protein 4 (BMP4) is required for migration and invasion of breast cancer. Mol Cell Biochem 363: 179-190, 2012.

7. Lian WJ, Liu G, Liu YJ, et al: Downregulation of BMP6 enhances cell proliferation and chemoresistance via activation of the ERK signaling pathway in breast cancer. Oncol Rep 30: 193-200, 2013

8. Rodriguez-Martinez A, Alarmo EL, Saarinen L, et al: Analysis of BMP4 and BMP7 signaling in breast cancer cells unveils time-dependent transcription patterns and highlights a common synexpression group of genes. BMC Med Genomics 4: 80, 2011.
9. Wang K, Feng H, Ren W, et al: BMP9 inhibits the proliferation and invasiveness of breast cancer cells MDA-MB-231. J Cancer Res Clin Oncol 137: 1687-1696, 2011.

10. Ye L, Bokobza S, Li J, et al: Bone morphogenetic protein-10 (BMP-10) inhibits aggressiveness of breast cancer cells and correlates with poor prognosis in breast cancer. Cancer Sci 101: 2137-2144, 2010.

11. You JS and Jones PA. Cancer genetics and epigenetics: two sides of the same coin? Cancer Cell 22: 9-20, 2012.

12. Kanwal R and Gupta S: Epigenetic modifications in cancer. Clin Genet 81: 303-311, 2012.

13. Wen XZ, Akiyama Y, Baylin SB, et al: Frequent epigenetic silencing of the bone morphogenetic protein 2 gene through methylation in gastric carcinomas. Oncogene 25: 2666-2673, 2006.

14. Fu B, Wang H, Wang J, et al: Epigenetic regulation of BMP2 by 1,25-dihydroxyvitamin D3 through DNA methylation and histone modification. PLoS One 8: e61423, 2013.

15. Livak KJ and Schmittgen TD: Analysis of relative gene expression data using real-time quantitative PCR and the 2(-Delta Delta C(T)) Method. Methods 25: 402-408, 2001.

16. Alarmo EL, Kuukasjärvi T, Karhu R, et al: A comprehensive expression survey of bone morphogenetic proteins in breast cancer highlights the importance of BMP4 and BMP7. Breast Cancer Res Treat 103: 239-246, 2007.

17. Davies SR, Watkins G, Douglas-Jones A, et al: Bone morphogenetic proteins 1 to 7 in human breast cancer, expression pattern and clinical/prognostic relevance. J Exp Ther Oncol 7: 327-338, 2008.

18. Ghosh-Choudhury N, Ghosh-Choudhury G, Celeste A, et al: Bone morphogenetic protein-2 induces cyclin kinase inhibitor p21 and hypophosphorylation of retinoblastoma protein in estradiol-treated MCF-7 human breast cancer cells. Biochim Biophys Acta 1497: 186-196, 2000.

19. Waite KA, Eng C: BMP2 exposure results in decreased PTEN protein degradation and increased PTEN levels. Hum Mol Genet 12: 679-684, 2003.

20. Clement JH, Raida M, Sänger J, et al: Bone morphogenetic protein 2 (BMP-2) induces in vitro invasion and in vivo hormone independent growth of breast carcinoma cells. Int J Oncol 27: 401-407, 2005.

21. Raida M, Clement JH, Leek RD, et al: Bone morphogenetic protein 2 (BMP-2) and induction of tumor angiogenesis. J Cancer Res Clin Oncol 131: 741-750, 2005.

22. Jones PA. Functions of DNA methylation: islands, start sites, gene bodies and beyond. Nat Rev Genet 13: 484-492, 2012.

23. De Carvalho DD, Sharma S, You JS, et al: DNA methylation screening identifies driver epigenetic events of cancer cell survival. Cancer Cell 21: 655-667, 2012.

24. Hervouet E, Cartron PF, Jouvenot M, et al: Epigenetic regulation of estrogen signaling in breast cancer. Epigenetics 8: 237-245, 2013.

25. Pang JM, Dobrovic A and Fox SB. DNA methylation in ductal carcinoma in situ of the breast. Breast Cancer Res 15: 206, 2013.

26. Connolly R and Stearns V: Epigenetics as a therapeutic target in breast cancer. J Mammary Gland Biol Neoplasia 17: 191-204, 2012.

27. Dagdemir A, Durif J, Ngollo M, et al: Breast cancer: mechanisms involved in action of phytoestrogens and epigenetic changes. In Vivo 27: 1-9, 2013.

28. Huang Y, Nayak S, Jankowitz R, et al: Epigenetics in breast cancer: what's new? Breast Cancer Res 13: 225, 2011. 